\title{
Descrição matemática do Método Flash para determinação da difusividade térmica.
}

\author{
Pinto, J. N. O.; Rouver, A. N.; Betini, E. G.; Correa, L. A.; Orlando, M. T. D. ${ }^{*}$; Passos, \\ C. A. ${ }^{1}$. \\ 1 Programa de Pós-Graduação em Engenharia Mecânica, Universidade Federal do Espírito Santo, Vitória, ES, Brasil. \\ *e-mail: julinop1988@gmail.com
}

\begin{abstract}
Resumo
Introduzido por Parker em 1961, o método do pulso de energia (Método Flash) é a mais usada e mais conhecida técnica para a determinação da difusividade térmica de materiais cerâmicos. Consiste de um método direto na determinação da difusividade térmica, no qual um pulso de energia radiante e de curta duração é incidido sobre uma das faces da amostra, sendo medida a variação de temperatura na face oposta. Este estudo tem o objetivo de descrever de forma explícita o caminho adotado por Parker para elaboração deste método. Partindo da equação de Difusão de calor, obtém-se a solução da equação diferencial parcial com condições de contorno não homogêneas, que fornece a relação da difusividade térmica com a largura da amostra e com o tempo para que o aumento de temperatura na face oposta a aplicação do feixe seja a metade do valor máximo.
\end{abstract}

\begin{abstract}
Introduced by Parker in 1961, the energy pulse method (Flash Method) is the most used technique for determination of thermal diffusivity of ceramic materials. It consists of a direct method of determining the thermal diffusivity, in which a radiant energy pulse of short duration is focused on one side of the sample, is measured temperature variation on the opposite side. This study aims to describe explicitly the path taken by Parker to prepare this method. From the heat diffusion equation obtains the solution to the partial differential equation with boundary conditions non-homogenous, which provides the ratio of thermal diffusivity to the width of the sample and the time for the temperature rise on the opposite face the beam application is half the maximum value.
\end{abstract}

Keywords: Flash Method, Thermal Diffusivity.

\section{Introdução}

A difusividade térmica tem considerável interesse em aplicações nas quais os materiais são expostos a altas temperaturas por um intervalo de tempo tão curto que o equilíbrio térmico sequer é atingido (choque térmico). Fisicamente a difusividade térmica é uma medida da rapidez com a qual o calor se propaga através de um material. Um material com difusividade térmica elevada responde mais rapidamente a mudanças de temperatura do que os materiais de menor difusividade térmica.

A difusividade térmica pode ser medida por métodos, de acordo com o regime de troca de calor utilizado, que se classificam em duas categorias [1]: Métodos estacionários - que medem a condutividade térmica num regime estacionário de troca de calor - e métodos não estacionários - medem a condutividade térmica durante um regime transiente de troca de calor.

Os métodos não estacionários podem ainda ser divididos em Método do Fluxo de Calor Periódico e Método do Fluxo de Calor Transiente. No primeiro, a fonte de calor faz variar a temperatura periodicamente numa das extremidades da amostra, sendo atingido o equilíbrio térmico após um determinado tempo e a difusividade térmica é determinada a partir de medidas de temperatura em diferentes pontos da amostra. Uma das desvantagens deste método está na exigência de um longo tempo para atingir as condições de equilíbrio. Outra desvantagem consiste no fato de que a temperatura ambiente precisa ser mantida constante durante a realização do experimento. No segundo método, é incidido sobre uma das faces da amostra um pulso de energia de curta duração. A difusividade é determinada a partir da medição do aumento de 
temperatura na face oposta a aplicação do laser. As principais vantagens deste método é que exige pouco tempo para realização das medidas, minimizando perdas de calor, além do uso de amostras com pequenas dimensões. A técnica experimental mais utilizada para realização do Método do Fluxo de Calor Transiente é o Método do Pulso de Energia introduzido por Parker [2].

\section{Método do Pulso de Energia (Método Flash)}

Introduzido por Parker em 1961 [2], é a mais usada e mais conhecida técnica para a determinação da difusividade térmica. Consiste de um método direto na determinação da difusividade térmica, onde um pulso de energia radiante e de curta duração é incidido sobre uma das faces da amostra, sendo medida a variação de temperatura na face oposta. Neste método, considera-se que o pulso de energia provoca um aumento de temperatura dentro da amostra suficientemente pequeno, para que se possam considerar as propriedades físicas constantes.

No trabalho de Parker, a amostra é aquecida através de um pulso de energia radiante, que consistia de uma lâmpada-flash de xenônio. Porém, as lâmpadas de xenônio foram substituídas com o aparecimento do laser. O motivo que levou a substituição das lâmpadas xenônio pelo laser foi devido ao feixe de laser ser monocromático, colimado e concentrar considerável energia em uma pequena área $[1,2]$.

\section{Descrição Matemática}

A maioria dos fenômenos de difusão ocorrem sob condições de regime transiente, ou seja, onde o fluxo de difusão e o gradiente em um ponto variam com o tempo. Neste caso, torna-se conveniente o uso da Equação de Difusão de Calor:

$$
\nabla^{2} T=\frac{1}{\alpha} \frac{\partial T}{\partial t}, \quad(0<x<l)
$$

onde $\alpha=\frac{k}{c \rho}$ e é a chamada difusividade térmica.

Considerando a amostra como uma placa plana infinita, a dimensão da direção de aplicação do pulso é muito menor que às outras coordenadas. Sendo assim, tratase do fluxo de calor unidirecional e perpendicular às faces paralelas da amostra, conforme ilustra a Figura 1.

Além disso, suponha que a temperatura inicial é dada por uma função $f(x)$ que descreve o pulso de energia incidindo sobre uma das faces e que ambos os contornos $x=0$ e $x=l$ são termicamente isolados, ou seja, são adiabáticos. Desse modo, tem-se:

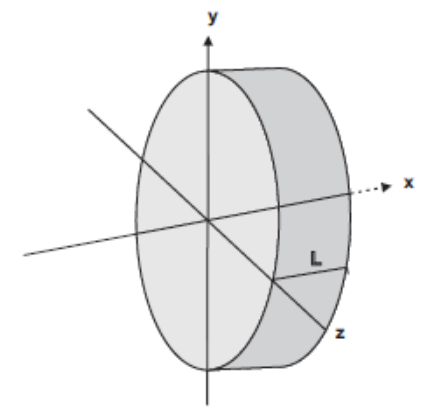

Figura 1: Orientação dos eixos com relação ao fluxo de calor.

$$
\begin{array}{lr}
\frac{\partial T}{\partial x}=0, & \text { quando } x=0 \text { e } x=l, \\
T(x, 0)=f(x), & \text { para } 0<x<l .
\end{array}
$$

Portanto, tem-se uma equação diferencial parcial com condições de contorno não homogêneas. Dessa maneira, é conveniente tratar o problema espacial sempre como um problema homogêneo, através do seguinte artifício:

$$
T(x, t)=v(x)+h(x, t)
$$

onde $v(x)$ atua como solução particular, que obedece as mesmas condições de contorno não homogêneas de $T(x, t)$. Assim, substituindo a equação (3) na equação (1), tem-se

$$
\frac{d^{2} v(x)}{d x^{2}}+\frac{\partial^{2} h(x, t)}{\partial x^{2}}=\frac{1}{\alpha} \frac{\partial h(x, t)}{\partial t} .
$$

Portanto, na equação (4) e com a condição homogênea, resulta que

$$
\frac{d^{2} v(x)}{d x^{2}}=0
$$

Logo,

$$
v=\text { Constante }=\frac{C_{0}}{2}
$$

futuramente ficarão mais claras as razões para escrever $v$ dessa forma.

Resolvendo (4) para $h(x, t)$, tem-se

$$
\frac{\partial^{2} h(x, t)}{\partial x^{2}}=\frac{1}{\alpha} \frac{\partial h(x, t)}{\partial t}
$$

Derivando parcialmente a equação (3) em relação a $x$, percebe-se que as condições de contorno de $h(x, t)$ são as mesmas de $T(x, t)$, ou seja, 


\section{Encontro Científico de Física Aplicada}

\section{Blucher}

$$
\frac{\partial h(x, t)}{\partial x}=0, \quad \text { quando } x=0 \text { e } x=l
$$

Aplicando separação de variáveis, tem-se:

$$
h(x, t)=X(x) \Lambda(t)
$$

Em (7), as funções $X(x)$ e $\Lambda(t)$ devem satisfazer as mesmas condições de contorno de $h(x, t)$, logo:

$$
\frac{d X(0)}{d x}=\frac{d X(l)}{d x}=0
$$

Substituindo (7) em (6), obtém-se:

$$
\frac{1}{X(x)} \frac{d^{2} X(x)}{d x^{2}}=\frac{1}{\alpha \Lambda(t)} \frac{d \Lambda(t)}{d t}=-\gamma^{2}
$$

Logo, para $x$, tem-se:

$$
\frac{d^{2} X(x)}{d x^{2}}=-\gamma^{2} X(x)
$$

que tem como solução

$$
X(x)=A \cos (\gamma x)+B \sin (\gamma x)
$$

Derivando a equação (10) e substituindo as condições de contorno (8), obtém-se o valor da constante $\gamma$ e se chega à solução da parte espacial da equação. Assim, para $x=0$, tem-se:

$$
\frac{d X(0)}{d x}=-A \cos (0)+B \sin (0)=B=0
$$

Para $x=l$,

$$
\frac{d X(l)}{d x}=-A \cos (\gamma l)=0,
$$

que, para solução não trivial $(A \neq 0)$, leva a

$$
X \gamma=\frac{n \pi}{l}
$$

Logo, a solução para a parte espacial da equação (9) será dada por

$$
X(x)=\operatorname{Acos}\left(\frac{n \pi}{l} x\right)
$$

Agora, resta encontrar a solução para a parte temporal, o que pode ser feito resolvendo a equação (9) para $t$ :

$$
\frac{d \Lambda(t)}{d t}=-\alpha\left(\frac{n \pi}{l}\right)^{2} \Lambda(t)
$$

cuja solução será:

$$
\Lambda(t)=e^{-\frac{\alpha n^{2} \pi^{2}}{l^{2}} t}
$$

Assim, $h(x, t)$ será o produto das soluções (11) e (12), conforme supôs-se em (7):

$$
h(x, t)=C \cos \left(\frac{n \pi}{l} x\right) e^{-\frac{\alpha n^{2} \pi^{2}}{l^{2}} t} .
$$

Portanto, agora que tanto $v(x)$ quanto $h(x, t)$ já foram determinadas, cabe voltar à equação (3) para encontrar a equação geral da (1). Logo, com (13) e (5), tem-se:

$$
T(x, t)=v(x)+h(x, t)=\frac{C_{0}}{2}+C \cos \left(\frac{n \pi}{l} x\right) e^{-\frac{\alpha n^{2} \pi^{2}}{l^{2}} t},
$$

que, usando a condição inicial em (2), reduz-se a:

$$
T(x, 0)=f(x)=\frac{C_{0}}{2}+\sum_{n=1}^{\infty} C_{n} \cos \left(\frac{n \pi}{l} x\right) .
$$

Perceba que esta é a Série de Fourier em cosseno de $f(x)$, com coeficiente $C_{n}$ definido como

$$
C n=\frac{2}{l} \int_{0}^{l} T(x, 0) \cos \left(\frac{n \pi}{l} x\right) d x
$$

Finalmente, substituindo essa expressão para $C_{n}$ na equação (14), chega-se a

$$
\begin{aligned}
& T(x, t)=\frac{1}{l} \int_{0}^{l} T(x, 0) d x+ \\
& +\frac{2}{l} \sum_{n=1}^{\infty} e^{-\frac{\alpha n^{2} \pi^{2}}{l^{2}} t} \cos \left(\frac{n \pi}{l} x\right) \int_{0}^{l} T(x, 0) \cos \left(\frac{n \pi}{l} x\right) d x .
\end{aligned}
$$

Outra consideração do Modelo de Parker é que no instante inicial $(t=0)$, o pulso de energia $Q$ é instantâneo e uniformemente absorvido em uma camada fina $g$ da superfície $(x=0)$ da amostra. Portanto,

$$
\begin{aligned}
T(x, 0) & =\frac{Q}{\rho c g}, \quad \text { se } 0<x<g, \\
& =0, \quad \text { se } g<x<l,
\end{aligned}
$$

onde $\rho$ é a densidade $\left(\mathrm{g} / \mathrm{cm}^{3}\right)$ do material e $c$ é o calor especifico $\left(\mathrm{cal} / \mathrm{g}^{\circ} \mathrm{C}\right)$. Aplicando essas condições iniciais na equação (15), segue que:

$T(x, t)=$

$\frac{Q}{\rho c g}\left[1+2 \sum_{n=1}^{\infty} \cos \left(\frac{n \pi}{l} x\right) e^{-\frac{\alpha n^{2} \pi^{2}}{l^{2}} t} \frac{l}{n \pi g} \sin \left(\frac{n \pi g}{l}\right)\right]$ 
Como a camada $g$ é pequena com relação à largura $l$ da amostra,

$$
\sin \left(\frac{n \pi g}{l}\right) \sim \frac{n \pi g}{l}
$$

Note também que, para $x=l$,

$$
\cos \left(\frac{n \pi}{l} x\right)=\cos (n \pi)=(-1) n \text {. }
$$

Então, com as duas substituições acima, a partir da equação (16) é fácil encontrar a função para o acréscimo de temperatura em $x=l$, dada por

$$
T(l, t)=\frac{Q}{\rho c l}\left[1+2 \sum_{n=1}^{\infty}(-1)^{n} e^{-\frac{\alpha n^{2} \pi^{2}}{l^{2}} t}\right] .
$$

É possível fazer $V(l, x)=T(l, x) / T_{\text {máx }}$, onde $T_{\text {máx }}=$ $\frac{Q}{\rho c l}$ é o máximo de acréscimo de temperatura em $x=l$ e também fazer $w=\left(\alpha \pi^{2} t\right) / l^{2}$, obtendo

$$
V(l, x)=1+2 \sum_{n=1}^{\infty}(-1)^{n} e^{-n^{2} w}
$$

Para um tempo $\tau$ necessário para que o aumento de temperatura na face da amostra oposta a aplicação do feixe seja a metade do valor máximo, ou seja, $V(l, x)=0,5$, encontra-se, através da equação (17), que $w=1,38$. Sendo assim, a difusividade pode finalmente ser determinada através do método do pulso de energia por meio da expressão a seguir:

$$
\alpha=\frac{1,38 l^{2}}{\pi^{2} t_{\frac{1}{2}}} .
$$

\section{Conclusão}

A Figura 2 ilustra a curva que se espera obter, medindo o aumento de temperatura na face oposta à aplicação do laser em função do tempo. A partir da mesma, a determinação da difusividade é feita, utilizando o tempo $\left(t_{1 / 2}\right)$ equivalente à metade do valor máximo para temperatura $(V=0,5)$.

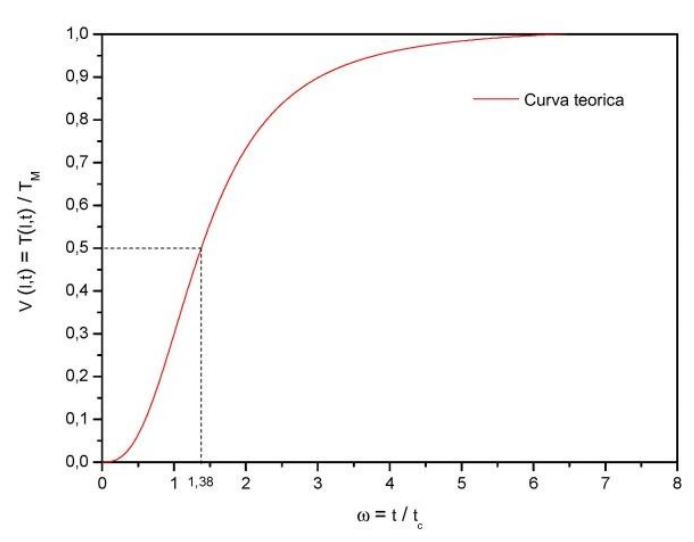

Figura 2: Curva experimental para a determinação da difusividade térmica [3].

Com isso, a difusividade térmica pode ser obtida, medindo-se a largura da amostra e o tempo para o qual o aumento de temperatura na face da amostra oposta a aplicação do feixe seja a metade do valor máximo.

Além disso, sabendo-se a densidade e o calor especifico do material, a condutividade térmica também pode ser encontrada de maneira indireta substituindo o valor encontrado para a difusividade térmica na equação, a saber:

$$
\alpha=\frac{k}{c \rho}
$$

a qual provém da equação de Condução de Calor.

\section{Agradecimentos}

Os autores agradecem ao Grupo de Física Aplicada pela oportunidade.

\section{Referências}

[1] SANTOS, W. N.; Cerâmica, v. 51, p. 205-213, 2005.

[2] PARKER, W. J. et al.; J. Appl. Phys., v. 32, p. 16791681, 1961.

[3] ROUVER, A. N. Determinação da influência do contorno de grão na medida de difusividade térmica em cerâmicas supercondutoras YBaCu3O7-x. 95f. Dissertação (Mestrado em Engenharia Mecânica), Programa de Pós-Graduação em Engenharia Mecânica, Universidade Federal do Espírito Santo, Vitória, 